\title{
Opposing Mechanisms Drive Richness Patterns of Core and Transient Bird Species
}

\author{
Jessica R. Coyle, ${ }^{1, \star}$ Allen H. Hurlbert, ${ }^{1}$ and Ethan P. White ${ }^{2}$ \\ 1. Department of Biology, CB 3280, University of North Carolina, Chapel Hill, North Carolina 27599; 2. Department of Biology and \\ the Ecology Center, Utah State University, Logan, Utah 84341
}

Submitted March 12, 2012; Accepted November 20, 2012; Electronically published March 8, 2013

Online enhancement: supplementary PDF. Dryad data: http://dx.doi.org/10.5061/dryad.q82nn.

\begin{abstract}
Studies of biodiversity typically assume that all species are equivalent. However, some species in a community maintain viable populations in the study area, while others occur only occasionally as transient individuals. Here we show that North American bird communities can reliably be divided into core and transient species groups and that the richness of each group is driven by different processes. The richness of core species is influenced primarily by local environmental conditions, while the richness of transient species is influenced primarily by the heterogeneity of the surrounding landscape. This demonstrates that the well-known effects of the local environment and landscape heterogeneity on overall species richness are the result of two sets of processes operating differentially on core and transient species. Models of species richness should focus on explaining two distinct patterns, those of core and transient species, rather than a single pattern for the community as a whole.
\end{abstract}

Keywords: birds, biodiversity, local environment, mass effects, occupancy, spatial heterogeneity, species richness.

\section{Introduction}

Understanding the distribution of biodiversity across the globe is a central goal of ecology, one that is of significant importance given impending threats to species (Wilcove et al. 1998; Pimm et al. 2006; Schipper et al. 2008) and potential range shifts due to global climate change and anthropogenic habitat alteration (Jetz et al. 2007; La Sorte and Jetz 2010). Traditionally, efforts to understand patterns of biodiversity have modeled the species richness of the community as a whole (Wright 1983; Rahbek and Graves 2001; White and Hurlbert 2010) even though it has long been suggested that species within a community can be divided into two distinct groups: (1) core species, which persist at a site through time, and (2) transient species,

* Corresponding author; e-mail: jrcoyle@live.unc.edu.

Am. Nat. 2013. Vol. 181, pp. 000-000. (C) 2013 by The University of Chicago. 0003-0147/2013/18104-53713\$15.00. All rights reserved.

DOI: $10.1086 / 669903$ which occur intermittently at a site as a result of dispersal from surrounding regions (Grinnell 1922; MacArthur 1960; Magurran and Henderson 2003). This core-transient distinction has provided important insights into community ecology because the processes governing the two groups differ, resulting in differences in ecological patterns. For example, core and transient species exhibit distinct forms of species abundance distribution (Magurran and Henderson 2003; Ulrich and Ollik 2004; Dolan et al. 2009), different levels of species turnover (Costello and Myers 1996), and different size distributions (Dolan et al. 2009).

The core-transient distinction is a temporal analog of the well-known core-satellite hypothesis (Hanski 1982) but differs in several important respects. The designation of a species as core or transient is specific to a local site, whereas under Hanski's framework, a species is labeled as core or satellite across an entire region. The primary approach for identifying core and transient species uses data on temporal occupancy instead of spatial occupancy. At a particular site, core species are those that are observed consistently through time, whereas transient species are observed erratically and infrequently (Costello and Myers 1996; Magurran and Henderson 2003; Ulrich and Ollik 2004; Vergnon et al. 2009). Narrowly distributed satellite species can still be "core" community members at the sites where they occur, and widely distributed species may be transient visitors to some of the sites at which they are observed; the core-transient distinction represents fundamentally different biological information than the coresatellite hypothesis, although linking these patterns further would be a productive area of research.

The implications of the core-transient distinction for understanding environmental drivers of species richness remain poorly explored. If the occurrence of core and transient species in local communities is mediated by different processes, then the two groups should differ substantially in the factors influencing their presence and abundance in ecological systems (Shmida and Wilson 
1985; Grime 1998; Novotný and Basset 2000; Magurran and Henderson 2003). Local environmental factors influencing the availability of niches and resources can strongly affect the number of species in a local community (MacArthur 1972; Wright 1983; Hurlbert 2004), as can regional factors that influence colonization dynamics (MacArthur and Wilson 1967; Ricklefs 1987). However, these factors will not affect core and transient species equally. The number of transient species at a local site should depend primarily on factors that increase the richness of potential immigrants, such as the heterogeneity of the surrounding landscape and the size of the regional species pool (Belmaker 2009; White and Hurlbert 2010). In contrast, if core species are those that maintain local populations at a site, then the number of core species should be more strongly tied to local environmental conditions that determine the suitability of the habitat for supporting populations of multiple species.

Despite this expected difference, we are aware of only one study exploring factors influencing the richness of core and transient species in natural systems. Belmaker (2009) found that the richness of the regional species pool was a stronger predictor of transient species richness than core species richness in coral reef fish. The lack of broad-scale studies examining species richness within a core-transient framework is in part due to the difficulty of characterizing species into the two groups, which requires either a detailed understanding of the biology of each species in the context of each habitat type (Belmaker 2009) or long-term data so that the distinction can be based on temporal occupancy (Magurran and Henderson 2003). We used data from one of the largest-scale long-term ecological monitoring programs available, the North American Breeding Bird Survey (BBS; North American Breeding Bird Survey 2011), to evaluate the extent to which North American breeding bird communities exhibit the core-transient species dichotomy and determine whether core and transient species richness patterns differ in a manner consistent with the different mechanisms proposed to explain core versus transient species occurrence.

\section{Methods}

We calculated core and transient species richness at all BBS (Bystrak 1981) sites surveyed every year from 1996 to 2010 (http://www.pwrc.usgs.gov/bbs accessed via EcoData Retriever 2011). Each survey consists of fifty 3-min point counts spaced evenly along a 39.4-km route, and we used only routes for which we could obtain spatial coordinates of the route's path (USGS Patuxent Wildlife Research Center 2012). We excluded species that are not well sampled by BBS methods, including raptors, nocturnal birds, and waterbirds (Robbins et al. 1986), resulting in a total of 336 species from 435 sites.

Richness was calculated as the total number of core or transient species present over a fixed time interval (19962010). However, core and transient species status was assigned using the proportion of years each species was present over all years the route was surveyed. Using the longest available time series provides the most accurate representation of site usage. We defined core species as those that were present for at least two-thirds of the years surveyed, while transient species were defined as those present in no more than one-third of the surveyed years. This approach attempts to minimize misclassification at the expense of excluding a small fraction of intermediate species from the analysis. We considered alternative occupancy thresholds, including the case of a single threshold (50\%), in which all species are classified and analyzed (appendix pt. A, available online).

To confirm that our results were not influenced by any of the other decisions made in our analyses, we conducted a series of sensitivity analyses. First, we calculated the proportion of species whose status shifted over the time period used to define core and transient species and verified that these shifts could not drive the patterns observed in our analyses of species richness (appendix pt. B, available online). Second, we performed analyses where the core versus transient status was determined over the same time period as the species richness (1996-2010; appendix pt. B). Third, we performed analyses in which richness was calculated across shorter time periods (appendix pt. B). All analyses produced results consistent with those presented.

We modeled core and transient species richness as a function of two classes of variables: those reflecting local environmental conditions and habitat quality (mean values of temperature and precipitation variables, a vegetation index) and those reflecting landscape heterogeneity (spatial variance in local variables, topography, and the size of the regional species pool). Spatial data layers for elevation and long-term average precipitation and temperature data were obtained from WorldClim (Hijmans et al. 2005) at 30" resolution. A long-term mean of summer (May-August) normalized difference vegetation index (NDVI) was calculated by averaging values from 2001 to 2010 using 1$\mathrm{km}$-resolution monthly composite vegetation indices from the USGS Land Processes Distributed Active Archive Center (https://lpdaac.usgs.gov/, product MOD13A3). Mean values of each environmental variable along the path of each route were used to characterize the local environment. The use of long-term means for characterizing local environmental variables focuses attention on average conditions at a site rather than on conditions in any particular year. Such year-to-year variability at any one site is small relative to the range of conditions spanned by this con- 
tinental analysis (e.g., the average range in NDVI at a site over 10 years is 0.067 , whereas the range in long-term mean NDVI across sites is 0.737 ). Landscape heterogeneity was characterized using the spatial variance of environmental variables within $40-\mathrm{km}$-radius buffers centered on the starting coordinates of each route path. We estimated regional species richness by counting the number of species range maps (Ridgely et al. 2003) overlapping each route's starting coordinates (White and Hurlbert 2010).

We evaluated univariate models relating core and transient species richness to each of the variables described above using ordinary least squares regression. All independent variables were normalized to $z$-scores so that effects could be compared across models. Spatial variance variables were highly skewed and were $\log _{\mathrm{e}}$ transformed prior to normalization. Because many of the environmental variables were strongly correlated with one another, we used ordination to generate independent variables for use in multivariate models of core and transient species richness. We conducted a principal components analysis (PCA) on 12 variables: six that characterized the local environment (summer NDVI, annual precipitation, precipitation of the wettest month, precipitation of the driest month, mean annual temperature, and maximum temperature) and six that reflected the spatial heterogeneity of the landscape (spatial variance in NDVI, annual precipitation, mean annual temperature, maximum temperature, and elevation, as well as mean elevation).

We used variance partitioning analyses (Legendre and Legendre 1998) to assess the unique contributions of local conditions and heterogeneity-related factors for predicting core and transient richness. Colinearity between predictors leads to a large fraction of nonuniquely ascribed variation in multivariate models, so we used two orthogonal variables from the PCA, each of which were shown to be related chiefly to either local environment or landscape heterogeneity (appendix pt. C, table S2, available online). We also used variance partitioning to evaluate the prediction that the richness of the regional species pool would be more important for explaining transient richness than core richness and the local environment would be more important for core richness than transient richness (Belmaker 2009; White and Hurlbert 2010).

Spatial autocorrelation in species richness may lead to an overestimation of the number of degrees of freedom in the analysis. We modeled richness at a site as conditional on the richness at neighboring sites using conditional autoregressive models (Fortin and Dale 2005) for the three multivariate models used in the variance partitioning and estimated parameters by maximum likelihood (function "spautolm" in the R library "spdep"; Bivand et al. 2011). Site adjacency was defined to decrease linearly to zero at a distance of $390 \mathrm{~km}$, ensuring no isolated sites.
To exclude the possibility that results were due to a distinction between abundant and rare species, as opposed to core and transient species, all analyses were also conducted on sets of species defined by their long-term abundance. Rare species were defined as those whose average abundance was less than 2.0 individuals in the years they were observed, and common species were defined as those with average abundance greater than 3.87. These definitions were chosen because they assigned approximately the same numbers of species to rare and common groups as were originally assigned to transient and core groups, thus maximizing the comparability of the two analyses (appendix pt. A).

\section{Results \\ Separation of Bird Communities into Core and Transient Species}

There was a clear distinction between core and transient species within local bird communities. The distributions of species' occurrence frequencies were bimodal at all but 28 of the 435 sites. The aggregate distribution across all sites was also strongly bimodal (fig. 1), with the majority of species $(83.6 \%)$ either occurring at a given site in most years $(51.9 \%$ core species $)$ or in just a few $(31.7 \%$ transient

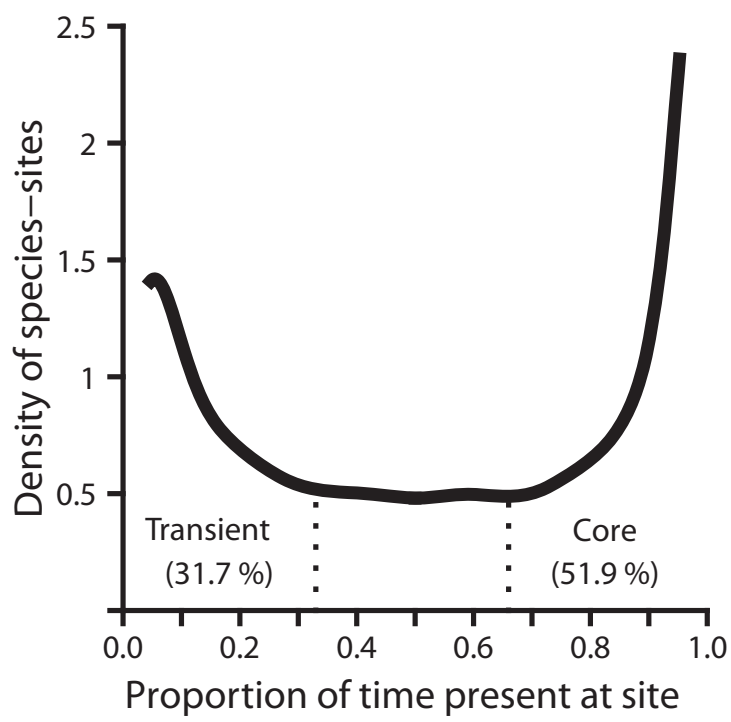

Figure 1: Probability density estimate of bird species' temporal occupancy across all 492 survey routes. The temporal occupancy of a species on a route is the proportion of surveyed years in which the species was recorded present. The solid line shows the relative frequency of each temporal occupancy level. Dotted lines indicate temporal occupancy levels used to classify species as core $(\geq 0.6667)$ or transient $(<0.3333) .51 .9 \%$ of all species across all routes $(n=$ $39,975)$ were core and $31.7 \%$ transient. 
species). Only a small fraction of species had intermediate occupancy.

\section{Univariate Analyses of Environmental Correlates}

Core and transient species richness display different geographic patterns (appendix pt. D, fig. S11, available online) and are not strongly correlated with each other $(r=$ $-0.10)$. Variables that measure local environmental conditions were more strongly and positively correlated with core richness, whereas variables associated with spatial heterogeneity were more strongly and positively correlated with transient richness (fig. 2, appendix pt. E, table S3, available online). In line with previous studies of North American birds (Hurlbert and Haskell 2003; White and Hurlbert 2010), NDVI was the strongest local environment predictor and explained $51 \%$ of the variance in core species richness. However, it had little effect on transient species richness, explaining less than $2 \%$ of the variance. This remotely sensed proxy for primary production during the avian breeding season may reflect both resource availability and vertical vegetation complexity, which are both expected to increase the equilibrial number of species that can be supported at a site (MacArthur and MacArthur 1961; Hurlbert 2004; Hurlbert and Jetz 2010).

\section{Multivariate Analyses of Environmental Correlates}

The first and second principal components of the PCA of 12 environmental variables encompassed $43.0 \%$ and $21.0 \%$ of the environmental variance, respectively. The loadings on these two components were consistent with an interpretation of the first component as primarily reflecting spatial heterogeneity and the second primarily reflecting the local environment (appendix pt. C, table S2). In variance partitioning analyses of core and transient species richness, the local environment component uniquely explained $28 \%$ of the variance in core species richness but less than $0.1 \%$ of the variance in transient richness. Conversely, the landscape heterogeneity component uniquely explained more than twice as much variance in transient richness as it did variance in core richness (fig. $3 A$ ).

Variance partitioning using two of the strongest univariate predictors (NDVI and elevational heterogeneity) indicates that while NDVI seems to be a negative predictor of transient richness based on univariate analyses, it has little effect once elevational heterogeneity is accounted for (fig. 3B). Variance partitioning using regional species richness and NDVI shows that the richness of the regional pool uniquely explained about five times more variance in transient richness than core richness and that NDVI uniquely explained about five times more variance in core richness than transient richness (fig. $3 C$ ).

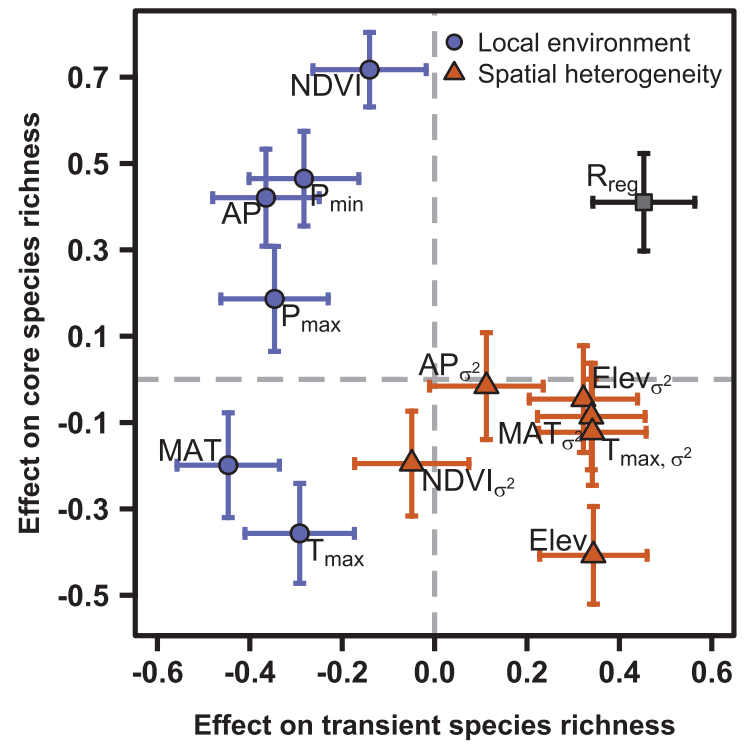

Figure 2: Parameter estimates for univariate models relating environmental variables and transient species richness versus core species richness. The position of each point represents the relative effect of each environmental variable on transient species richness versus core species richness. Blue circles denote variables measuring local environmental conditions, and orange triangles denote variables measuring spatial heterogeneity. The gray square reflects the effects of an independently calculated measure of regional species richness. $\mathrm{AP}=$ annual precipitation, Elev = elevation, $\mathrm{MAT}=$ mean annual temperature, NDVI = normalized difference vegetation index, $P_{\max }=$ precipitation in the wettest month, $P_{\min }=$ precipitation in the driest month, $R_{\text {reg }}=$ regional species richness, and $T_{\max }=$ maximum yearly temperature. A subscript of $\sigma^{2}$ indicates that the spatial variance of the specified variable was used. Error bars show $99 \%$ confidence intervals for coefficient estimates.

\section{Robustness of Results}

Using different occupancy thresholds and determining species richness over shorter time spans had little effect on our results (appendix pts. A and B). Estimated regression coefficients did not differ between spatial and nonspatial models (appendix pt. E, table S4), and estimated autocorrelation parameters were small in all models $(\leq 0.029)$. Fifty-eight species occurred infrequently across all sites where they were observed and were not classified as core at any site. These species are likely to be classified as transient even on sites where they maintain continuous populations because they are either rare or difficult to detect. However, excluding these species did not influence our results (appendix pt. A). Analyses based on rare and common species groups produced different results from the core-transient analysis. In contrast to transient richness, rare species richness was uncorrelated with elevation but positively correlated with NDVI $(r=0.23)$, while the 

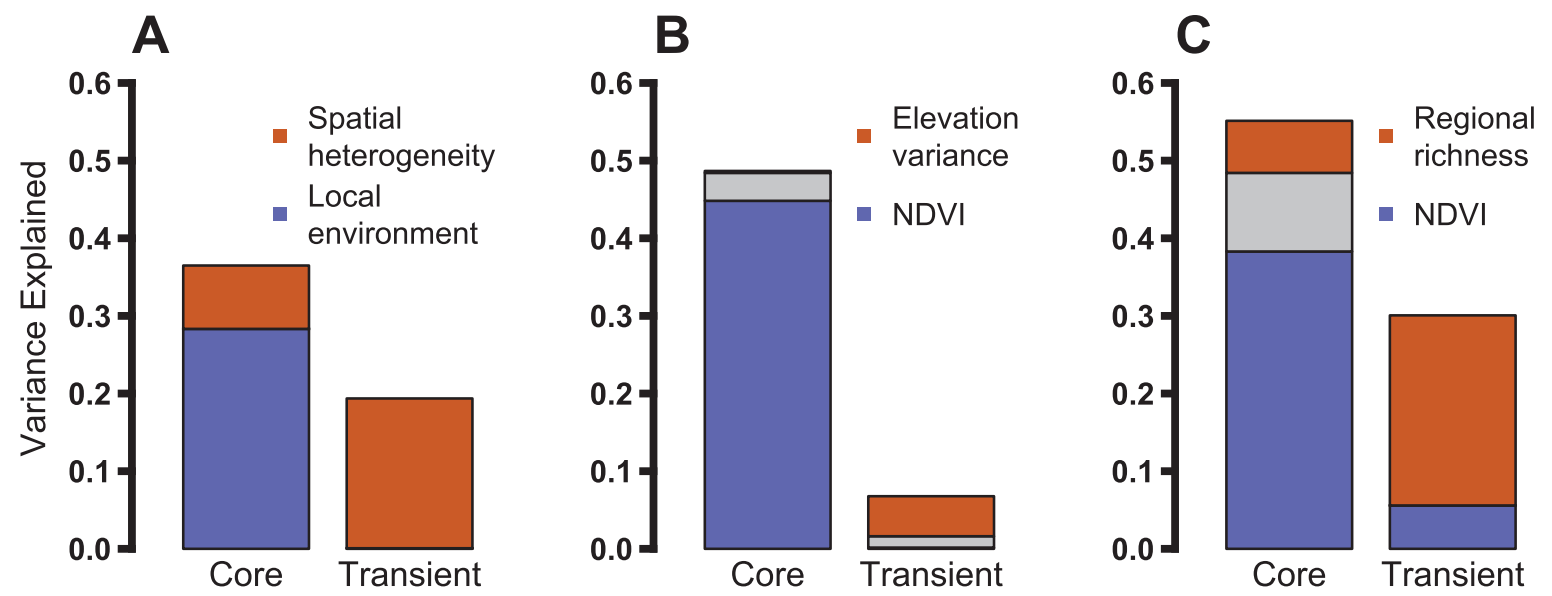

Figure 3: Variance of core and transient species richness partitioned between two classes of variables. Orange bars show the proportion of variance in species richness that can be uniquely attributed to regional factors. Blue bars show the proportion of variance uniquely attributed to local factors. Gray bars show variance explained by both factors. A, The spatial heterogeneity and local environment variables are the first and second principal components from a PCA of the 12 environmental variables in figure 2. Models containing only elevational variance and summer NDVI are shown in B. C represents a comparison of summer NDVI and an independently calculated measure of richness of the regional species pool.

correlations between common species richness and local environmental variables were all weaker than the respective correlations with core species richness (appendix pt. A, figs. S1, S3).

\section{Discussion}

The core-transient framework makes four predictions: (1) a positive relationship between transient species richness regional-scale spatial heterogeneity, (2) a positive relationship between core richness and local-scale environment, (3) no relationship between transient richness and local-scale environment, and (4) no relationship between core richness and regional-scale spatial heterogeneity. Both univariate and multivariate analyses are consistent with all four of these predictions, suggesting that local- and landscape-level factors have opposite effects on core versus transient species richness.

The differences between the regression models of core and transient species richness reflect the fact that the two groups arise in communities through different mechanisms, which is consistent with at least one mathematical model (Schwilk and Ackerly 2005). Core richness is strongly influenced by local-scale factors, because in order to maintain local populations, species must successfully compete in the abiotic and biotic environment, coping with local stressors and potential resource constraints. Transient richness is strongly influenced by regional factors that govern the number of species that could potentially immigrate into the focal habitat from the surrounding landscape. In North America, mountainous areas of high heterogeneity tend to be of lower productivity, and thus the mechanisms that drive core and transient richness on this continent oppose one another (appendix pt. D, fig. S11). This leads to situations in which the richness of different local communities reaches the same level through different mechanisms. For example, a community of 75 species in the North Carolina Piedmont is composed of $69 \%$ core species and $9 \%$ transient species, whereas a community of 75 species in the Colorado Rockies has $49 \%$ core species and $36 \%$ transient species (appendix pt. F). The similarity in overall species richness between these two sites belies a potentially fundamental difference in effective diversity. In general, long-term species richness of local sites in the mountains involves a strong influence of mass effects (Shmida and Wilson 1985) that are enhanced by the spatial heterogeneity of the region, whereas richness in relatively homogeneous regions will depend more on the productivity of those regions. The linear effect of regional richness on transient richness is consistent with the idea that transient richness does not saturate with local environmental conditions but rather is limited by the supply of potential colonists (Belmaker 2009). This pattern also suggests that ecological theory related to the importance of the regional pool for determining species richness is more relevant to the subset of transient species rather than to the diversity of the system as a whole.

We have identified two groups of species that respond 
differently to environmental gradients in a manner consistent with that predicted if one group were limited by local environmental conditions and the other were limited by regional factors. Nevertheless, we recognize several challenges in accurately identifying members of these groups and in ensuring that the distinction between core and transient species is not simply a reflection of some alternative distinction such as commonness and rarity. Species that occur at very low densities or have poor detectability may erroneously be recorded as absent on a given survey, and therefore some fraction of the species categorized as transients may in fact be regularly present. The critical issue for this study is whether this fraction varies systematically with environmental gradients in a manner that might generate observed patterns. For birds, detectability is generally lower in more mature deciduous forest with greater foliage density (Schieck 1997; Pacifici et al. 2008), and therefore the effect of this bias should result in an overestimation of the number of transient species in regions of high NDVI and high deciduous forest cover. However, transient richness is uncorrelated with the proportion of deciduous forest cover $(r=0.019$; USGS 2001 National Land Cover Database, Homer et al. 2007) and negatively correlated with NDVI $(r=-0.14)$, making it unlikely that such detectability biases drive the observed relationships. Given that NDVI and spatial heterogeneity are negatively correlated, a detectability bias is similarly unlikely to generate the observed positive relationship between transient richness and spatial heterogeneity. Finally, the misclassifications of core versus transient species should be independent of the richness of the regional pool, and rare species are expected to be more prevalent in high productivity settings (Hubbell 2001; Bonn et al. 2004), both of which should weaken the relationships in figure 3.

Several studies have decomposed communities into common and rare species and shown that the determinants of richness may differ for the two groups (Lennon et al. 2004, 2011). The identification of core and transient species in the BBS data is correlated with common and rare ones, but imperfectly so, and the common-rare distinction yields richness relationships that are weaker than those obtained using the core-transient dichotomy (appendix pt. A, table S1). Furthermore, the bimodal nature of the occupancy distribution makes the assignment into core and transient groups more straightforward than the distinction between common and rare based on a unimodal abundance distribution (appendix pt. A, fig. S4). Most importantly, the biology of core and transient species makes four clear predictions that are all supported, whereas predictions for how the importance of the local environment and regional heterogeneity should vary as a function of abundance alone are less clear. Based on these observa- tions, we have confidence that the results presented here reflect an important distinction between core and transient species that is neither artifactual nor a surrogate for commonness versus rarity. Nevertheless, any study attempting to identify core and transient species from temporal occupancy data alone must carefully consider these critical issues of distinguishing rare from transient species and exploring how gradients in species detectability might influence observed relationships.

Studies of species richness that do not account for the differences between core and transient species may reach inaccurate conclusions about the role of diversity in ecosystems, especially in areas of high heterogeneity such as mountain ranges, ecotones, and island archipelagoes. The reasons for this are threefold. First, transient species are not functionally equivalent to core species because transient species usually occur at low abundance and, by definition, are infrequently present in the community (Dolan et al. 2009; Gaston 2010). Our results may explain findings by other investigators that the richness of rare bird species is not well predicted by available energy (Evans et al. 2005, 2006), if rare bird species are more likely to be categorized as transient. Second, core and transient species may interact with components of the community differently, because core and transient species do not experience the same ecological and evolutionary pressures. Unlike core species, the population genetics and dynamics of transient species are largely unaffected by ecological interactions in locations where they are transient, because the bulk of their population is located elsewhere. Because of this, core and transient species are likely to respond differently to environmental change. Third, theoretical models of species richness tend to be based on processes that are either primarily local or primarily regional in nature. Since the proportions of core and transient species vary geographically (appendix pt. D, fig. S12) and will likely also vary among taxonomic groups and across spatial scales, support for any particular theory may depend on what fraction of the community is made up of core versus transient species, rather than on differences in the operation of the underlying processes.

Despite Joseph Grinnell's treatise on the "role of the 'accidental' " 90 years ago (Grinnell 1922), the role of transient species in ecological communities has received little attention. Since transient species comprise a large proportion of the overall species richness in some communities, more attention to their unique role is clearly warranted. Of particular interest in the face of ongoing climate and habitat change are the potentially different responses of core and transient species to perturbations, where transient species may serve as important reservoirs of potential colonists to facilitate the maintenance of both diversity and ecosystem function in the future (Grinnell 1922; 
Grime 1998; Magurran and Henderson 2003; Dolan et al. $\rightarrow$ Gaston, K. J. 2010. Valuing common species. Science 327:154-155. 2009).

$\rightarrow$ Grime, J. P. 1998. Benefits of plant diversity to ecosystems: immediate, filter and founder effects. Journal of Ecology 86:902-910.

$\rightarrow$ Grinnell, J. 1922. The role of the "accidental." Auk 39:373-380.

$\rightarrow$ Hanski, I. 1982. The core and satellite species hypothesis. Oikos 38: 210-221.

\section{Acknowledgments}

We thank the many volunteers and staff associated with the North American Breeding Bird Survey for collecting, cleaning, and hosting the bird data set and the developers of EcoData Retriever (http://ecologicaldata.org/ecodata -retriever) for facilitating its download and use. We thank D. McGlinn and J. Stegen for providing feedback on the research and resulting manuscript and two anonymous reviewers for comments that significantly improved the manuscript. E.P.W. was supported by a grant from the National Science Foundation (DEB-0953694). J.R.C. received support from the Royster Society of Fellows at the University of North Carolina.

\section{Literature Cited}

$\rightarrow$ Belmaker, J. 2009. Species richness of resident and transient coraldwelling fish responds differentially to regional diversity. Globa ${ }^{1}$ Ecology and Biogeography 18:426-436.

Bivand, R., M. Altman, L. Anselin, R. Assuncao, O. Berke, A. Bernat, G. Blanchet, et al. 2011. spdep: spatial dependence, weighting schemes, statistics and models. R package version 0.5-40. http:/! cran.r-project.org/web/packages/spdep/index.html.

$\rightarrow$ Bonn, A., D. Storch, and K. J. Gaston. 2004. Structure of the speciesenergy relationship. Proceedings of the Royal Society B: Biological Sciences 271:1685-1691.

Brown, J. H. 1995. Macroecology. University of Chicago Press. Chicago.

Bystrak, D. 1981. The North American Breeding Bird Survey. Studies in Avian Biology 6:34-41.

$\rightarrow$ Costello, M. J., and A. A. Myers. 1996. Turnover of transient species as a contributor to the richness of a stable amphipod (Crustacea) fauna in a sea inlet. Journal of Experimental Marine Biology and Ecology 202:49-62.

$\rightarrow$ Dolan, J. R., M. E. Ritchie, A. Tunin-Ley, and M.-D. Pizay. 2009. Dynamics of core and occasional species in the marine plankton: tintinnid ciliates in the north-west Mediterranean Sea. Journal of Biogeography 36:887-895.

EcoData Retriever. Ecological data wiki. Accessed January 27, 2011. http://ecologicaldata.org/ecodata-retriever.

$\rightarrow$ Evans, K. L., J. J. Greenwood, and K. J. Gaston. 2005. Relative contribution of abundant and rare species to species-energy relation ships. Biology Letters 1:87-90.

$\rightarrow$ Evans, K. L., N. A. James, and K. J. Gaston. 2006. Abundance, species richness and energy availability in the North American avifauna. Global Ecology and Biogeography 15:372-385.

$\rightarrow$ Farnsworth, G. L., K. H. Pollock, J. D. Nichols, T. R. Simons, J. E. Hines, and J. R. Sauer. 2002. A removal model for estimating detection probabilities from point-count surveys. Auk 119:414425.

Fortin, M. J., and M. R. T. Dale. 2005. Spatial analysis: a guide for ecologists. Cambridge University Press, Cambridge. $\rightarrow$ Hijmans, R. J., S. E. Cameron, J. L. Parra, P. G. Jones, and A. Jarvis. 2005. Very high resolution interpolated climate surfaces for global land areas. International Journal of Climatology 25:1965-1978.

Homer, C., J. Dewitz, J. Fry, M. Coan, N. Hossain, C. Larson, N. Herold, et al. 2007. Completion of the 2001 National Land Cover Database for the conterminous United States. Photogrammetric Engineering and Remote Sensing 73:337-341.

Hubbell, S. P. 2001. The unified neutral theory of biodiversity and biogeography. Princeton University Press, Princeton, NJ.

Hurlbert, A. H. 2004. Species-energy relationships and habitat complexity in bird communities. Ecology Letters 7:714-720.

$\rightarrow$ Hurlbert, A. H., and J. P. Haskell. 2003. The effect of energy and seasonality on avian species richness and community composition. American Naturalist 161:83-97.

$\rightarrow$ Hurlbert, A. H., and W. Jetz. 2010. More than "more individuals": the nonequivalence of area and energy in the scaling of species richness. American Naturalist 176:E50-E65.

$\rightarrow$ Jetz, W., D. S. Wilcove, and A. P. Dobson. 2007. Projected impacts of climate and land-use change on the global diversity of birds. PLoS Biology 5:e157.

Kennedy, C. M., P. P. Marra, W. F. Fagan, and M. C. Neel. 2010. Landscape matrix and species traits mediate responses of Neotropical resident birds to forest fragmentation in Jamaica. Ecological Monographs 80:651-669.

$\rightarrow$ La Sorte, F. A., and W. Jetz. 2010. Avian distributions under climate change: towards improved projections. Journal of Experimental Biology 213:862-869.

Legendre, P., and L. Legendre. 1998. Numerical ecology. 2nd ed. Elsevier, Amsterdam.

$\rightarrow$ Lennon, J. J., C. M. Beale, C. L. Reid, M. Kent, and R. J. Pakeman. 2011. Are richness patterns of common and rare species equally well explained by environmental variables? Ecography 34:529-539.

$\rightarrow$ Lennon, J. J., P. Koleff, J. J. D. Greenwood, and K. J. Gaston. 2004. Contribution of rarity and commonness to patterns of species richness. Ecology Letters 7:81-87.

$\rightarrow$ MacArthur, R. 1960. On the relative abundance of species. American Naturalist 94:25-36.

MacArthur, R. H. 1972. Geographical ecology. Princeton University Press, Princeton, NJ.

$\rightarrow$ MacArthur, R. H., and J. W. MacArthur. 1961. On bird species diversity. Ecology 42:594-598.

MacArthur, R. H., and E. O. Wilson. 1967. The theory of island biogeography. Princeton University Press, Princeton, NJ.

$\rightarrow$ Magurran, A. E., and P. A. Henderson. 2003. Explaining the excess of rare species in natural species abundance distributions. Nature 422:714-716.

Mattsson, B. J., and M. R. Marshall. 2009. Occupancy modeling as a framework for designing avian monitoring programs: a case study along Appalachian streams in southern West Virginia. Pages 617-632 in Proceedings of the Fourth International Partners in Flight Conference: Tundra to Tropics. McAllen, TX.

North American Breeding Bird Survey. USGS Patuxent Wildlife Research Center. Accessed January 27, 2011. http://www.pwrc.usgs .gov/bbs/. 
$\rightarrow$ Novotný, V., and Y. Basset. 2000. Rare species in communities of tropical insect herbivores: pondering the mystery of singletons. Oikos 89:564-572.

$\rightarrow$ Pacifici, K., T. R. Simons, and K. H. Polluck. 2008. Effects of vege tation and background noise on the detection process in auditory avian point-count surveys. Auk 125:600-607.

$\rightarrow$ Pimm, S., P. Raven, A. Peterson, Ç. H. Şekercioğlu, and P. R. Ehrlich. $\rightarrow$ 2006. Human impacts on the rates of recent, present, and future bird extinctions. Proceedings of the National Academy of Science of the USA 103:10941-10946.

$\rightarrow$ Preston, F. W. 1962. The canonical distribution of commonness and rarity. I. Ecology 43:185-215.

R Development Core Team. 2011. R: a language and environment for statistical computing. R Foundation for Statistical Computing, Vienna. http://www.R-project.org/.

$\rightarrow$ Rahbek, C., and G. R. Graves. 2001. Multiscale assessment of patterns of avian species richness. Proceedings of the National Academy or Sciences of the USA 98:4534-4539.

$\rightarrow$ Ricklefs, R. E. 1987. Community diversity: relative roles of local and regional processes. Science 235:167-171.

Ridgely, R. S., T. F. Allnutt, T. Brooks, D. K. McNicol, D. W. Mehlman, B. E. Young, and J. R. Zook. 2003. Digital distribution maps of the birds of the Western Hemisphere. Ver. 1. NatureServe, Arlington, VA. http://www.natureserve.org/getData/birdMaps.jsp.

Robbins, C. S., D. Bystrak, and P. H. Geissler. 1986. The breeding bird survey: its first fifteen years, 1965-1979. US Fish and WildlifeService, Washington, DC.

$\rightarrow$ Schieck, J. 1997. Biased detection of bird vocalizations affects comparisons of bird abundance among forested habitats. Condor 99: 179-190.

$\rightarrow$ Schipper, J., J. S. Chanson, F. Chiozza, N. A. Cox, M. Hoffmann, V.
Katariya, J. Lamoreux, et al. 2008. The status of the world's land and marine mammals: diversity, threat, and knowledge. Science 322:225-230.

$\rightarrow$ Schwilk, D. W., and D. D. Ackerly. 2005. Limiting similarity and functional diversity along environmental gradients. Ecology Letters 8:272-281. doi:10.1111/j.1461-0248.2004.00720.x.

Shmida, A., and M. V. Wilson. 1985. Biological determinants of species diversity. Journal of Biogeography 12:1-20.

$\rightarrow$ Ulrich, W., and M. Ollik. 2004. Frequent and occasional species and the shape of relative-abundance distributions. Diversity and Distributions 10:263-269.

USGS Patuxent Wildlife Research Center. 2012. The North American Breeding Bird Survey, route geographic information summaries 1966-2003. Ver. 2004.1. USGS Patuxent Wildlife Research Center, Laurel, MD. http://www.mbr-pwrc.usgs.gov/bbs/geographic _information/geographic_information_products_htm.

$\rightarrow$ Vergnon, R., N. K. Dulvy, and R. P. Freckleton. 2009. Niches versus neutrality: uncovering the drivers of diversity in a species-rich community. Ecology Letters 12:1079-1090.

$\rightarrow$ White, E. P., and A. H. Hurlbert. 2010. The combined influence of the local environment and regional enrichment on bird species richness. American Naturalist 175:E35-E43.

$\rightarrow$ Wilcove, D. S., D. Rothstein, J. Dubow, A. Phillips, and E. Losos. 1998. Quantifying threats to imperiled species in the United States. BioScience 48:607-615.

$\rightarrow$ Wright, D. H. 1983. Species-energy theory: an extension of speciesarea theory. Oikos 41:496-506.

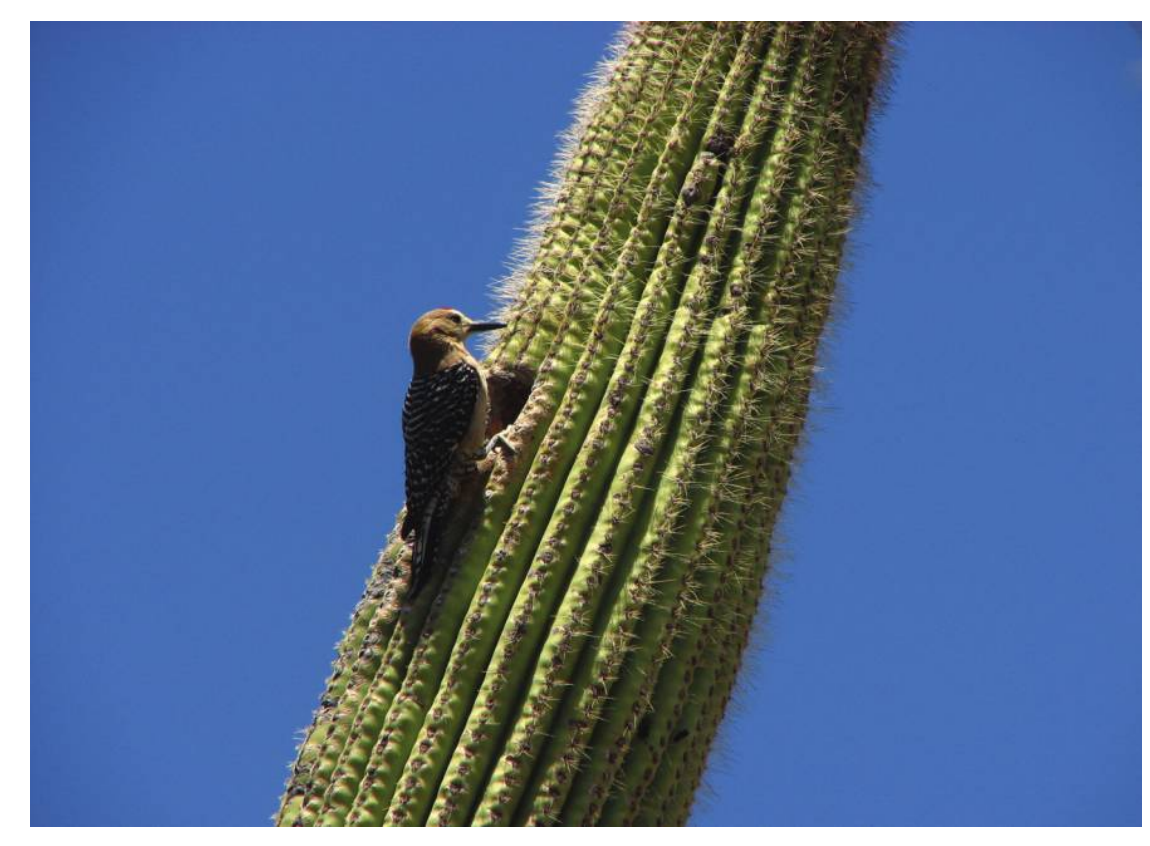

A male gila woodpecker (Melanerpes uropygialis) has excavated a nest in a Saguaro cactus near Tucson, Arizona. A resident of the Sonoran Desert, this species has been recorded on four of the Breeding Bird Survey routes analyzed as a part of this study: twice as a core species and once as a transient species. Photograph by J. R. Coyle et al. 